\title{
Trapped waves between submerged obstacles
}

\author{
By F. DIA $S^{1}$ AND J.-M. VANDEN-BROECK ${ }^{2}$ \\ ${ }^{1}$ Centre de Mathématiques et de Leurs Applications, Ecole Normale Supérieure de Cachan, 61 av \\ Président Wilson, 94235 Cachan, France \\ ${ }^{2}$ School of Mathematics, University of East Anglia, Norwich, UK
}

(Received 18 December 2003 and in revised form 16 March 2004)

Free-surface flows past submerged obstacles in a channel are considered. The fluid is assumed to be inviscid and incompressible and the flow to be irrotational. In previous work involving a single obstacle (Dias \& Vanden-Broeck 2002), new solutions called 'generalized hydraulic falls' were found. These solutions are characterized by a supercritical flow on one side of the obstacle and a train of waves on the other. However, in the case of a single submerged object, the generalized hydraulic falls are unphysical because the waves do not satisfy the radiation condition. In this paper new solutions for the flow past two obstacles of arbitrary shape are computed. These solutions are characterized by a train of waves 'trapped' between the obstacles. The generalized hydraulic falls are shown to describe locally the flow over one of the two obstacles when the distance between the two obstacles is large.

\section{Introduction}

Free-surface potential flows generated by disturbances moving at a constant velocity in a channel of finite depth are considered. The disturbances can be a pressure distribution with bounded support, a surface-piercing object (i.e. a ship) or a submerged object. We take a frame of reference moving with the disturbance(s) and seek steady solutions. These solutions also describe the free-surface flows past one or several fixed disturbances in a current. The results presented in this paper are qualitatively independent of the nature of the disturbances.

Using weakly nonlinear theories and fully nonlinear computations, Dias \& VandenBroeck (2002) showed that in the case of a single obstacle there are four basic types of solutions in which the flow is required to be uniform in the far field. Due to the reversibility of potential flows, this uniform stream can be either at the front or the back of the disturbance. We introduce Cartesian coordinates with the $x$-axis parallel to the bottom and assume without loss of generality that the uniform stream occurs as $x \rightarrow \infty$. We define the Froude number

$$
F=\frac{U}{(g H)^{1 / 2}},
$$

where $U$ and $H$ are the constant velocity and depth as $x \rightarrow \infty$ and $g$ is the acceleration due to gravity. When the flow is also uniform as $x \rightarrow-\infty$, we introduce in addition the Froude number

$$
F^{*}=\frac{U^{*}}{\left(g H^{*}\right)^{1 / 2}}
$$


(a)

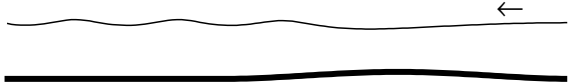

(c)

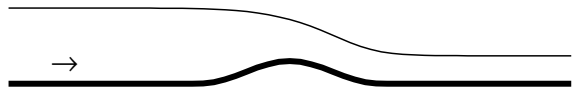

(b)

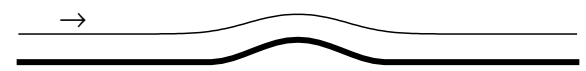

FIGURE 1. The four basic free-surface flows past a single submerged obstacle: $(a)$ Subcritical uniform flow upstream and waves downstream. (b) Supercritical flow on both sides. (c) Subcritical flow upstream and supercritical flow downstream (hydraulic fall). (d) Wavy subcritical flow upstream and supercritical flow downstream (generalized hydraulic fall). The horizontal scale is the same as the vertical scale. All solutions are computed solutions based on the full incompressible Euler equations with a free surface. Since the flows are reversible, the arrows which indicate the direction of the flow can in principle be reversed.

Here $U^{*}$ and $H^{*}$ are the constant velocity and depth as $x \rightarrow-\infty$. The uniform flow at infinity is called supercritical if $F>1$ and subcritical if $F<1$. Similarly the flow as $x \rightarrow-\infty$ (when uniform) is called supercritical if $F^{*}>1$ and subcritical if $F^{*}<1$.

Solutions of the first type have a subcritical flow as $x \rightarrow \infty$ (i.e. $F<1$ ) and a train of waves as $x \rightarrow-\infty$ (see figure $1 a$ ). Solutions of the second type have uniform supercritical flows both upstream and downstream and are characterized by $F=F^{*}>1$ (see figure $1 b$ ). The third type of solution (see figure $1 c$ ) is a hydraulic fall with a uniform subcritical flow as $x \rightarrow-\infty$ (i.e. $F^{*}<1$ ) and a uniform supercritical flow as $x \rightarrow \infty$ (i.e. $F>1$ ). The fourth type of solution (see figure $1 d$ ) is similar to the third but has a train of waves as $x \rightarrow-\infty$. The first three types of solution are classical and have been studied by many previous investigators (see for example Lamb 1932; Forbes \& Schwartz 1982; Vanden-Broeck 1988; Forbes 1988; Dias \& Vanden-Broeck $1989,2002)$. Solutions of the fourth type are relatively new and have only been calculated in the fully nonlinear regime by Dias \& Vanden-Broeck (2002). Dias \& Vanden-Broeck called this fourth type of flow a generalized hydraulic fall to contrast it with the classical hydraulic fall (third type of solution) which is characterized by a uniform stream upstream.

Potential flows are reversible and the direction of the arrows in figure 1 can in principle be reversed. This is, for example, the case for the flow of figure 1(b). However when waves are present on the free surface, physically relevant solutions have to satisfy the radiation condition. This condition, which requires that there is no energy coming from infinity, implies that waves can only occur on the downstream portion on the flow. The direction of the flow (i.e. the direction of the arrow) has been chosen in figure $1(a)$ so that the radiation condition is satisfied. However the flow of figure $1(d)$ does not satisfy the radiation condition. One might attempt to resolve this difficulty by reversing the direction of the arrow. Unfortunately hydraulic falls are only observed with subcritical flow upstream, i.e. with the orientation of the arrows shown in figure $1(c, d)$ (see for example the monograph by Viollet et al. 1998). In conclusion, the physical relevance of the flow of figure $1(d)$ is limited in the case of a single submerged obstacle.

Here, as in the rest of the paper, a frame of reference moving with the obstacles is chosen and the flows are assumed to be steady. Figure 2 shows a flow past two submerged obstacles with a train of waves 'trapped' between the obstacles. The flow is 
(a)

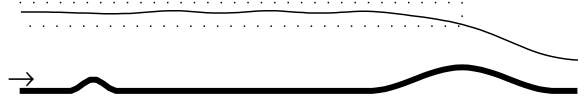

(b)

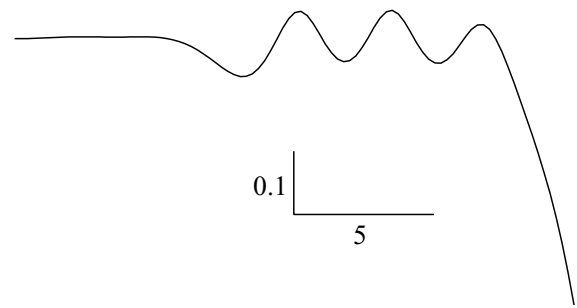

Figure 2. An example of a flow past two submerged obstacles: (a) Global picture (the horizontal scale is the same as the vertical scale). (b) Blow-up of the region in the dotted box. The uniform flow is subcritical upstream. The uniform flow is supercritical downstream. Waves are trapped between the obstacles.

uniform as $x \rightarrow-\infty$ so that the radiation condition is now satisfied. Solutions similar to the one sketched in figure 2 have not been calculated before in the fully nonlinear regime. Pratt (1984) performed experiments with two obstacles and interpreted them in the framework of a weakly nonlinear model. He observed several types of steady solutions. In particular, he found solutions with a regular train of waves between the obstacles, the flow being uniform and subcritical upstream of the first obstacle and uniform and supercritical downstream of the second obstacle (see the sketch in Pratt's figure $4 a$ and the photograph of an experimental flow in Plate 1). Pratt also found that only the details of the wave amplitudes and lengths depend on obstacle shape. In the present paper we provide conclusive numerical evidence of the existence of solutions with trapped waves between the obstacles.

The portion of the flow of figure 2 on top of the second obstacle is similar to the one shown in figure $1(d)$. The portion of the flow on top of the first obstacle is similar to the one shown in figure $1(a)$ with $x$ replaced by $-x$. We show below that these two portions of the flow of figure 2 approach those of figures $1(a)$ and $1(d)$ in the limit as the distance between the obstacles tends to infinity.

The problem of figure 2 is formulated in $\S 2$, the numerical procedure is described in $\S 3$ and the numerical results are discussed in $\S 4$.

\section{Formulation}

The flow configuration of figure 2 is considered. It is bounded below by a horizontal bottom, except for the presence of one or several bumps, and above by a free surface. The fluid is assumed to be inviscid and incompressible and the flow to be irrotational. We introduce Cartesian coordinates with the $x$-axis along the flat part of the bottom and the $y$-axis directed vertically upwards. Gravity is acting in the negative $y$ direction. As $x \rightarrow \infty$, the flow approaches a uniform stream with constant velocity $U$ and constant depth $H$. We define dimensionless variables by choosing $U$ as the unit velocity and $H$ as the unit length. The Froude number (1.1) is assumed to be greater than 1 , so that the flow is supercritical as $x \rightarrow \infty$. The problem is formulated in terms of the velocity potential $\phi(x, y)$. This function satisfies Laplace's equation

$$
\phi_{x x}+\phi_{y y}=0
$$

in the flow domain with the boundary conditions

$$
\begin{gathered}
\phi_{x}^{2}+\phi_{y}^{2}+\frac{2}{F^{2}} y=1+\frac{2}{F^{2}} \quad \text { on } \quad y=\eta(x), \\
\phi_{y}=\phi_{x} \eta_{x} \quad \text { on } \quad y=\eta(x),
\end{gathered}
$$




$$
\begin{gathered}
\phi_{y}=\phi_{x} B_{x} \text { on } y=B(x), \\
\phi_{x} \rightarrow 1, \quad \eta(x) \rightarrow 1 \quad \text { as } x \rightarrow \infty .
\end{gathered}
$$

Here $y=\eta(x)$ is the (unknown) equation of the free surface and $y=B(x)$ is the (prescribed) equation of the bottom. Equation (2.2) is the dynamic boundary condition derived from Bernoulli's equation and (2.3) and (2.4) are the kinematic boundary conditions on the free surface and on the bottom respectively. The condition (2.5) forces a uniform flow at infinity with velocity and depth both equal to 1 . This concludes the formulation of the problem. We seek two functions $\phi(x, y)$ and $\eta(x)$ satisfying (2.1) together with the boundary conditions (2.2)-(2.5). One of the difficulties encountered in solving this type of problem is to find the number $P$ of independent dimensionless parameters needed to determine a solution uniquely. It was shown by the analytical and numerical work of Dias \& Vanden-Broeck (2002) and others that $P$ depends on the flow under study. In order to understand the importance of the number of parameters $P$, one can rely on experiments. Imagine that the obstacles have given shapes and sizes. Then two obvious parameters that one can vary are the speed of the towed obstacle and the water depth. Intuitively, one expects to find a unique solution for each pair of speed and depth (or equivalently each pair of Froude number and dimensionless obstacle size). And indeed $P=2$ for the flows of figure $1(a)$ and $1(b)$. But $P=1$ for the flow of figure $1(c)$ and $P=3$ for the flow of figure $1(d)$.

\section{Numerical procedure}

The problem is first reformulated as a system of integro-differential equations by following the work of Forbes \& Schwartz (1982), Belward \& Forbes (1993), Forbes (1988) and Dias \& Vanden-Broeck (2002). The free surface is described parametrically by $x=X(s)$ and $y=Y(s)$, where $s$ is the arclength. Therefore we require

$$
X^{\prime 2}(s)+Y^{\prime 2}(s)=1,
$$

where the prime denotes derivative with respect to $s$. We choose $s=0$ at the point $x=0$ on the free surface (i.e. $X(0)=0$ ). Following Belward \& Forbes (1993), we derive the integral equations

$$
\begin{gathered}
-\pi\left[\phi^{\prime}(s) X^{\prime}(s)-\gamma\right]=\int_{-\infty}^{\infty} \frac{\left[\phi^{\prime}(\sigma)-\gamma X^{\prime}(\sigma)\right][Y(\sigma)-Y(s)]-\gamma[X(\sigma)-X(s)] Y^{\prime}(\sigma)}{[X(\sigma)-X(s)]^{2}+[Y(\sigma)-Y(s)]^{2}} \mathrm{~d} \sigma \\
+\int_{-\infty}^{\infty} \frac{\gamma B^{\prime}(\sigma)[\sigma-X(s)]+[B(\sigma)-Y(s)]\left\{\bar{u}(\sigma)\left[1+B^{\prime}(\sigma)^{2}\right]-\gamma\right\}}{[\sigma-X(s)]^{2}+[B(\sigma)-Y(s)]^{2}} \mathrm{~d} \sigma
\end{gathered}
$$

and

$$
\begin{gathered}
-\pi[\bar{u}(x)-\gamma]=\int_{-\infty}^{\infty} \frac{\left[\phi^{\prime}(\sigma)-\gamma\right][Y(\sigma)-B(x)]-\gamma[X(\sigma)-x] Y^{\prime}(\sigma)}{[X(\sigma)-x]^{2}+[Y(\sigma)-B(x)]^{2}} \mathrm{~d} \sigma \\
+\int_{-\infty}^{\infty} \frac{\gamma B^{\prime}(x)[\sigma-x]+[B(\sigma)-B(x)]\left\{\bar{u}(\sigma)\left[1+B^{\prime}(\sigma)^{2}\right]-\gamma\right\}}{[\sigma-x]^{2}+[B(\sigma)-B(x)]^{2}} \mathrm{~d} \sigma,
\end{gathered}
$$

where $\phi(s)$ is the velocity potential on the free surface and $\bar{u}(x)$ the horizontal component of the velocity on the bottom. The first integral in (3.2) and the second integral in (3.3) are Cauchy principal values. Equations (3.2) and (3.3) are obtained by applying the Cauchy integral equation formula to the function $\phi_{x}-\gamma-\mathrm{i} \phi_{y}$ with a contour consisting of the free surface, the bottom of the channel and two vertical 
lines at $x=-\infty$ and $x=\infty$. The constant $\gamma$ is chosen as the undisturbed velocity as $x \rightarrow-\infty$ (a precise definition is given later).

Next the dynamic boundary condition (2.2) is rewritten as

$$
\left[\phi^{\prime}(s)\right]^{2}+\frac{2}{F^{2}}[Y(s)-1]=1 .
$$

This concludes the reformulation of the problem as a system of integro-differential equations. We seek four unknown functions $X(s), Y(s), \phi(s)$ and $\bar{u}(x)$ satisfying (3.1)-(3.4). To solve these equations numerically we introduce the $N$ mesh points

$$
s_{I}=-\frac{(N-1) E}{2}+(I-1) E, \quad I=1, \ldots, N,
$$

on the free surface and the $M$ mesh points

$$
x_{I}=-\frac{(M-1) h}{2}+(I-1) h, \quad I=1, \ldots, M,
$$

on the bottom. The intervals of the discretization are $E$ and $h$. We also use the midpoints

$$
s_{I}^{m}=\frac{s_{I}+s_{I+1}}{2}, \quad I=1, \ldots, N-1,
$$

and

$$
x_{I}^{m}=\frac{x_{I}+x_{I+1}}{2}, \quad I=1, \ldots, M-1 .
$$

For clarity we describe the numerical scheme for the flow configurations of figures $1(c)$ and $1(d)$. The length of the disturbance is denoted by $2 L_{2}$ and its maximum thickness by $2 A_{2}$. The appropriate changes to compute the flow of figure 2 will be described in $\S 4$. The $3 N+M+3$ unknowns are $2 A_{2}, F, \gamma, X_{I}^{\prime}=X^{\prime}\left(s_{I}\right), Y_{I}^{\prime}\left(s_{I}\right), \phi^{\prime}\left(s_{I}\right), I=1, \ldots, N$ and $\bar{u}\left(x_{I}\right), I=1, \ldots, M$. In all the calculations it is assumed that $L_{2}$ (and $L_{1}$ in the case of two obstacles) is (are) given.

We satisfy (3.2) at the mesh points $s_{I}^{m}$. The integrals are approximated by the trapezoidal rule with a summation over the mesh points $s_{I}$ and $x_{I}$. The symmetry of the quadrature and of the discretization enables us to evaluate the Cauchy principal value integral (first integral in (3.2)) as if it were an ordinary integral. This leads to $N-1$ algebraic equations. Similarly $M-1$ algebraic equations are obtained by satisfying (3.3) at the mesh points $x_{I}^{m}$. The next $2 N$ equations are derived by satisfying (3.1) and (3.4) at the mesh points $s_{I}$. Two more equations are obtained by imposing

$$
\bar{u}\left(x_{M}\right)=1
$$

and

$$
\gamma^{2}+\frac{2}{\gamma F^{2}}-1-\frac{2}{F^{2}}=0 .
$$

Relation (3.9) forces a uniform stream with velocity 1 as $x \rightarrow \infty$ while relation (3.10) defines the undisturbed velocity as $x \rightarrow-\infty$.

We have now $3 N+M$ equations with $3 N+M+3$ unknowns. Three more equations are needed. As explained at the end of $\S 2$, these last three equations depend on the flow under study. For the flow of figure 1(c) $(P=1)$, these three equations are defined as follows. The free surface is forced to be flat as $x \rightarrow-\infty$ by imposing the conditions

$$
Y_{1}^{\prime}=Y_{2}^{\prime}=0
$$




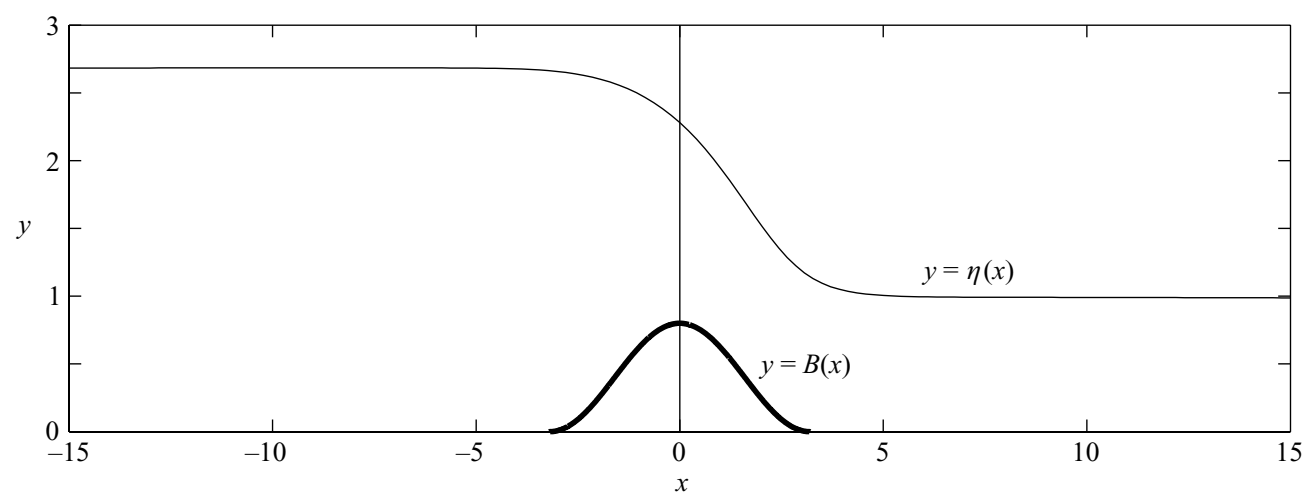

FIgURE 3. Computed hydraulic fall past a single submerged obstacle $\left(A_{1}=0\right)$ in the shape of the square of a cosine. The obstacle is characterized by $A_{2}=0.4$ and $L_{2}=3.2$. The Froude number is found as part as the solution: $F=1.977$.

and the value of $A_{2}$ is fixed. For the flow of figure $1(d)(P=3)$, the three extra equations are defined by fixing values of $F, A_{2}$ and by imposing

$$
Y\left(s_{1}\right)=a,
$$

where $a$ is prescribed. Equation (3.12) fixes the value of $Y$ at the first mesh point. This condition does not have a physical meaning but is a convenient numerical way to fix the third parameter. After a solution has been computed, it can be characterized by the amplitude of the waves instead of the parameter $a$. For a given value of $L_{2}$, we now have a system of $3 N+M+3$ nonlinear algebraic equations with $3 N+M+3$ unknowns. This system is solved by Newton iterations.

\section{Numerical results}

The numerical computations were performed by assuming that the obstacles have 'cosine' profiles. Therefore we write

$$
\left.\begin{array}{l}
B(x)=2 A_{1} \cos ^{2}\left(\frac{\pi(x+l)}{2 L_{1}}\right), \quad-L_{1}<x+l<L_{1}, \\
B(x)=2 A_{2} \cos ^{2}\left(\frac{\pi x}{2 L_{2}}\right), \quad-L_{2}<x<L_{2}, \\
B(x)=0, \quad \text { otherwise, }
\end{array}\right\}
$$

where $A_{1}, A_{2}, l, L_{1}$ and $L_{2}$ are prescribed constants for all the flows we consider. When $A_{1} \neq 0$ and $A_{2}=0$ or $A_{1}=0$ and $A_{2} \neq 0,(4.1)$ corresponds to a single obstacle. When $A_{1} \neq 0$ and $A_{2} \neq 0,(4.1)$ describes two obstacles separated by the distance $l$. In (4.1), the origin $x=0$ is chosen at the centre of the second obstacle.

We first describe results for one obstacle $\left(A_{2} \neq 0, A_{1}=0\right)$. These results are qualitatively similar to those previously obtained for triangular obstacles by Dias \& Vanden-Broeck (1989) and for circular obstacles by Forbes (1988), Vanden-Broeck (1988) and Dias \& Vanden-Broeck (2002). Typical profiles are shown in figures 3 and 4. Figure 3 shows a hydraulic fall for $L_{2}=3.2, A_{2}=0.4$ and the (unknown) Froude number $F=1.977$. The flow is supercritical as $x \rightarrow \infty$ and subcritical as $x \rightarrow-\infty$. There are no waves on the free surface. For a given value of $L_{2}$, the hydraulic falls define a one-parameter family of solution $(P=1)$. This means that solutions are 

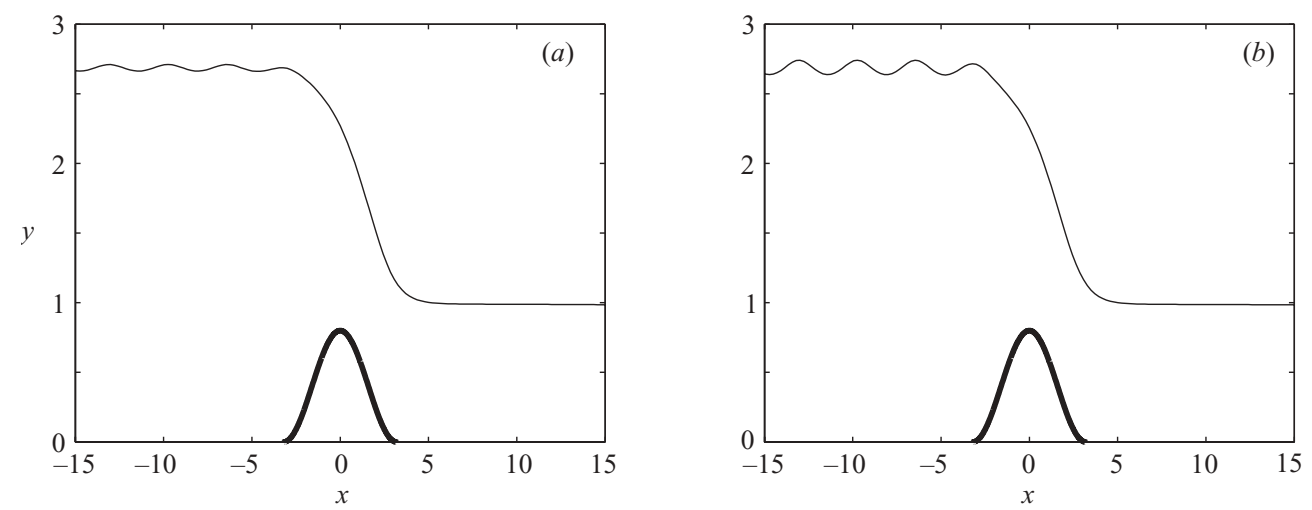

FIGURE 4. Computed generalized hydraulic fall past a single submerged obstacle $\left(A_{1}=0\right)$ in the shape of the square of a cosine. The obstacle is characterized by $A_{2}=0.4$ and $L_{2}=3.2$. The prescribed Froude number is $F=1.977$. Plots $(a)$ and $(b)$ differ by the amplitude of the waves, which is prescribed.

uniquely defined by fixing one parameter, for example $A_{2}$. The value of the Froude number $F$ is then found as part of the solution. Figure 4 shows some generalized hydraulic falls. This type of solution was first computed by Dias \& Vanden-Broeck (2002) in the fully nonlinear case. For a given value of $L_{2}$, these solutions form a three-parameter family of solutions $(P=3)$. This means that a solution is uniquely defined by fixing three parameters, for example $A_{2}, F$ and the amplitude of the waves on the free surface.

Solutions with two obstacles similar to the one of figure 2 have been observed experimentally by Pratt (1984). In his experiments, the obstacles are fixed and water is circulated through the flume by a variable-speed pump. The choice (4.1) for the shape of the obstacles agrees fairly well with the experimental set up of Pratt. In a frame of reference moving with the obstacles (or in the laboratory frame of reference in the case of Pratt's experiments), the flow is in the direction of the arrow of figure 2. The generalized hydraulic falls of figure 4 have not been observed experimentally in the case of one obstacle. This is consistent with the fact that the waves in figure 4 do not satisfy the radiation condition (which requires that there is no supply of energy from infinity). It is shown below that the generalized hydraulic falls of figure 4 describe locally free-surface flows with waves trapped between the obstacles.

Typical free-surface flows past two obstacles $\left(A_{1} \neq 0, A_{2} \neq 0\right)$ are shown in figures $5(a)$ and $5(b)$. These solutions were obtained by using the scheme of $\S 3$, with $A_{1}, A_{2}$ and $l$ fixed. This means that we prescribe values of $L_{1}, L_{2}, A_{1}$ and $l$ in (4.1) and define the last three equations (see discussion at the end of $\S 3$ ) by fixing $A_{2}$ and imposing (3.11). The flow is uniform and supercritical as $x \rightarrow \infty$ and uniform and subcritical as $x \rightarrow-\infty$. There is a train of waves trapped between the obstacles. Since there are no waves in the far field the radiation condition is satisfied. As the distance $l$ between the obstacles increases, the flow on top of the obstacle centred at $x=0$ approaches the generalized hydraulic fall past a single obstacle (see examples in figure 4). This is shown explicitly in figures $5(c)$ and 6 . Figure $5(c)$ shows a solution with trapped waves. Figure 6 shows a generalized hydraulic fall which agrees with the portion of flow of figure 5(c) near $x=0$. This solution was computed by assigning to $F$ and $A_{2}$ the same values as those for the flow of figure 5(c) and adjusting the value of $a$ in (3.12) so that 

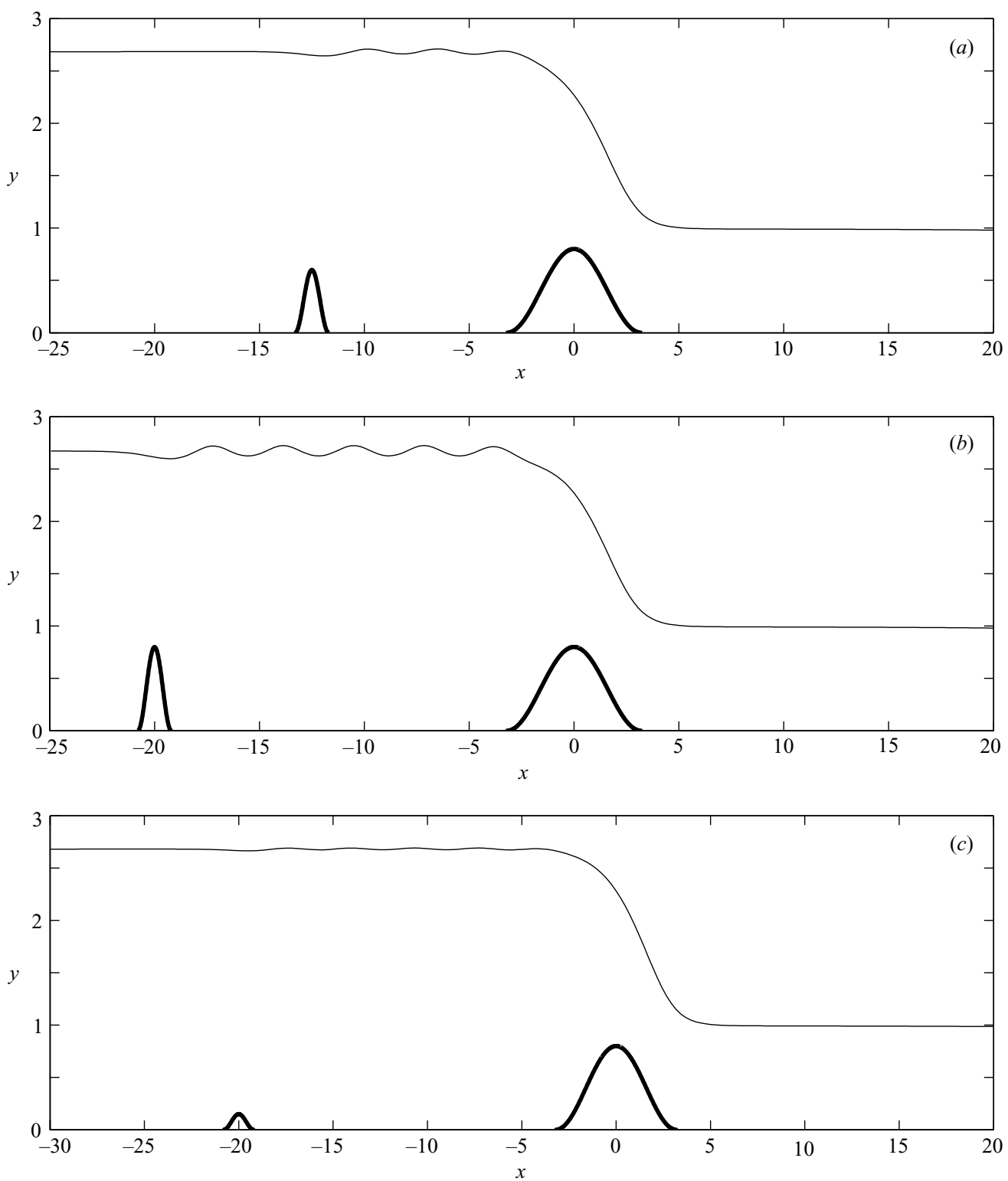

FIGURE 5. Computed hydraulic fall past two submerged obstacles in the shape of the square of a cosine. Waves are trapped between the obstacles. The obstacles are characterized by $A_{2}=0.4, L_{1}=0.8, L_{2}=3.2$, and $(a) A_{1}=0.3, l=12.5 ;(b) A_{1}=0.4, l=20 ;(c) A_{1}=0.075$, $l=20$. The Froude number is found as part of the solution: $(a) F=1.976,(b) F=1.971$, (c) $F=1.976$.

the waves in figure 6 have the same amplitude as in figure 5(c). An argument based on weakly nonlinear theory can be given to convince the reader that the flow of figure 5(c) indeed approaches the generalized hydraulic fall of figure 6. Dias \& Vanden-Broeck (2002) showed that under some assumptions (long waves, small bump), the flow past an obstacle can be described by the forced Korteweg-de Vries equation

$$
\eta_{x x}+\frac{9}{2} \eta^{2}-6\left(F^{*}-1\right) \eta=-3 B(x),
$$




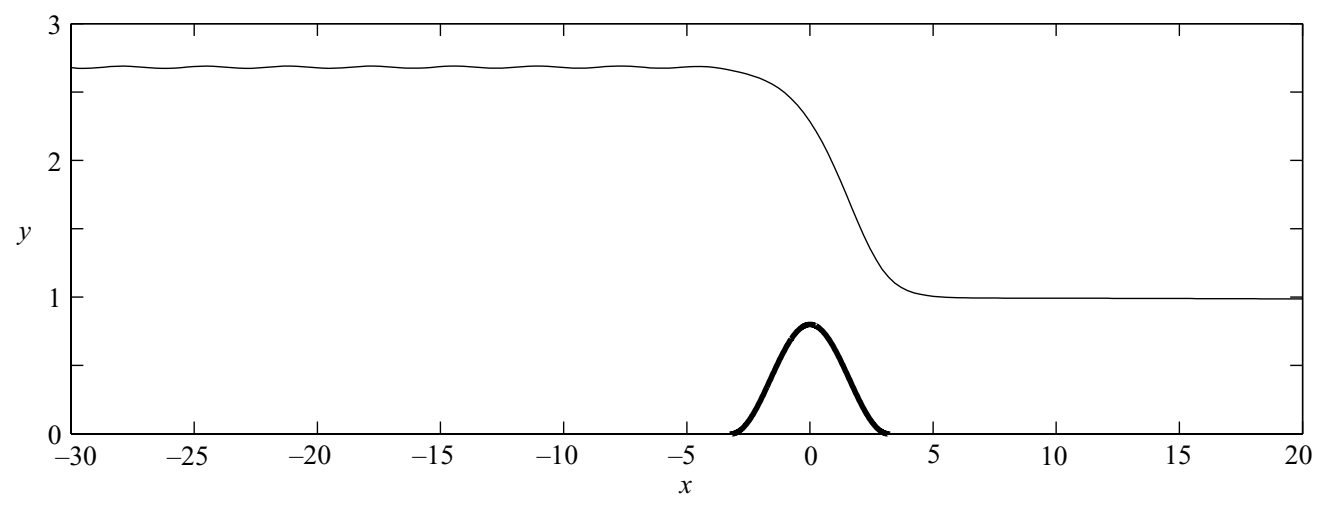

FIGURE 6. Computed generalized hydraulic fall past a single submerged obstacle $\left(A_{1}=0\right)$ in the shape of the square of a cosine. The obstacle is characterized by $A_{2}=0.4$ and $L_{2}=3.2$. The prescribed Froude number is $F=1.976$.

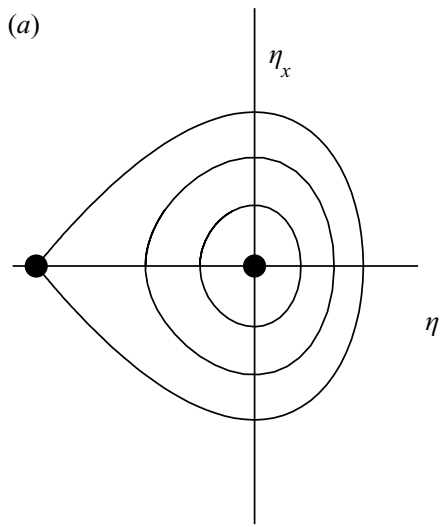

(a)

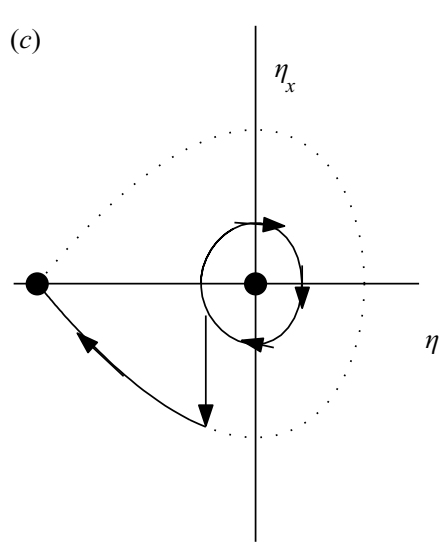

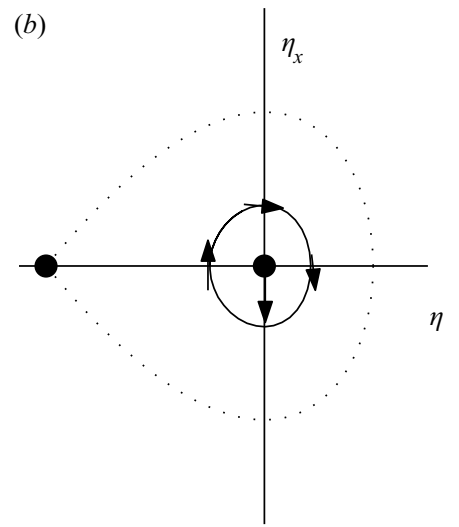

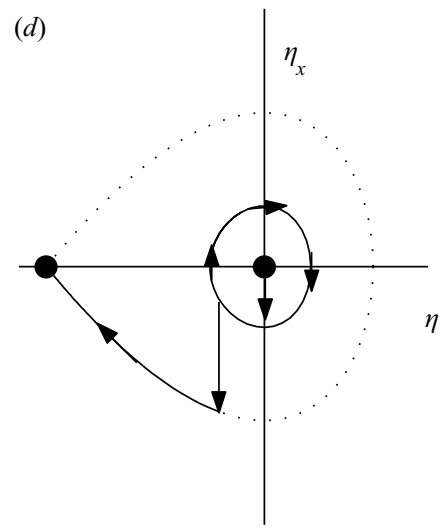

FiguRE 7. Phase planes corresponding to the forced Korteweg-de Vries equation (4.2). The fixed points are indicated. (a) Unforced bounded solutions. (b) Trajectory corresponding to the subcritical flow of figure $1(a)$ with $x$ replaced by $-x$. There is a negative jump in $\eta_{x}$ from the origin to the periodic wave. (c) Trajectory corresponding to the flow of figure 6 . There is a negative jump in $\eta_{x}$ from the periodic wave to the solitary wave. $(d)$ Trajectory corresponding to the flow of figure $5(c)$. The phase plane $(d)$ simply is a superposition of the phase planes (b) and $(c)$. There are two jumps in $\eta_{x}$, one from the origin to the periodic wave and one from the periodic wave to the solitary wave. 
where $F^{*}$ is the Froude number (1.2) and $\eta$ the deviation from the water level at $x=-\infty$. For obstructions whose height is comparable with the length of the obstruction base ('local' forcing), the forcing can be approximated by the Dirac delta function in the dimensionless long-wave coordinates. Then the problem reduces to seeking solutions which are continuous and bounded for all $x$, and satisfy

$$
\eta_{x x}+\frac{9}{2} \eta^{2}-6\left(F^{*}-1\right) \eta=0 \quad \text { for } \quad x \neq x_{\text {bump }}, \quad \eta_{x}\left(x_{\text {bump }}^{-}\right)-\eta_{x}\left(x_{\text {bump }}^{+}\right)=3 Q,
$$

where $x_{\text {bump }}$ denotes the location of the bump and $Q$ the bump size. The jump condition is obtained by integrating (4.2) from $x_{\text {bump }}^{-}$to $x_{\text {bump }}^{+}$. In the presence of two obstacles, there are two jumps, say at $x=x_{\text {bump }}^{1}$ and $x=x_{\text {bump }}^{2}$. Figure 7 shows four phase planes: one corresponding to the unforced equation (4.2), one corresponding to the flow of figure $1(a)$ with $x$ replaced by $-x$, one corresponding to the flow of figure $1(d)$ (or 6), and one corresponding to the flow of figure 5(c). Without forcing, the non-trivial bounded solutions are either cnoidal waves going around the origin $\eta=0, \eta_{x}=0$, or a solitary wave (figure $7 a$ ). In the subcritical flow of figure $1(a)$ with $x$ replaced by $-x$, the solution is uniform for $x<0(\eta=0)$ and it is a cnoidal wave for $x>0$ (figure $7 b$ ). In the supercritical flow of figure 6 , the solution is a cnoidal wave for $x<0$ and it follows the solitary wave solution for $x>0$ (figure $7 c$ ). In the two-obstacle flow of figure 5(c), the solution is uniform for $x<x_{\text {bump }}^{1}(\eta=0)$, a cnoidal wave for $x_{\text {bump }}^{1}<x_{\text {bump }}^{2}$ and a solitary wave for $x>x_{\text {bump }}^{2}$ (figure $7 d$ ). Increasing the distance between obstacles merely increases the number of waves without changing the lengths or amplitudes of the existing waves, as already noticed by Pratt (1984). The above description generalizes to forcings which are not necessarily Dirac delta functions, thus confirming that the flow on top of the second obstacle is a generalized hydraulic fall while the flow on top of the first obstacle is a subcritical wavy flow.

This work was partially supported by EPSRC, the National Science Foundation (NSF) and Alliance (the Franco-British Joint Research Programme of the British Council, with Project Number 05697WF).

\section{REFERENCES}

Belward, S. R. \& Forbes, L. K. 1993 Fully non-linear two-layer flow over arbitrary topography. J. Engng Maths 27, 419-432.

Dias, F. \& VANDEN-BroecK, J.-M. 1989 Open channel flows with submerged obstructions. J. Fluid Mech. 206, 155-170.

Dias, F. \& Vanden-Broeck, J.-M. 2002 Generalised critical free-surface flows. J. Engng Maths 42, 291-301.

Forbes, L. K. 1988 Critical free-surface flow over a semi-circular obstruction. J. Engng Maths 22, $3-13$.

Forbes, L. K. \& Schwartz, L. W. 1982 Free-surface flow over a semicircular obstruction. J. Fluid Mech. 114, 299-314.

Lamb, H. 1932 Hydrodynamics, 6th edn. Cambridge University Press.

Pratt, L. J. 1984 On nonlinear flow with multiple obstructions. J. Atmos. Sci. 41, 1214-1225.

VANDEN-BroecK, J.-M. 1988 Free-surface flow over a semi-circular obstruction in a channel. Phys. Fluids 30, 2315-2317.

Viollet, P.-L., Chabard, J.-P., Esposito, P. \& Laurence, D. 1998 Mécanique des Fluides Appliquée. Presses de l'Ecole Nationale des Ponts et Chaussées. 